\title{
MICROBIAL DYSBIOSIS AND MICROBIOTA- GUT-RETINA AXIS: THE LESSON FROM BRAIN NEURODEGENERATIVE DISEASES TO PRIMARY OPEN-ANGLE GLAUCOMA PATHOGENESIS OF AUTOIMMUNITY
}

\author{
NARTtaya Chaiwiang and TeERa PoyomtiP* \\ Faculty of Optometry, Ramkhamhaeng University, Bangkok, Thailand
}

(Received: 23 March 2019; accepted: 23 April 2019)

\begin{abstract}
In recent years, microbiota-associated neurodegenerative diseases have been exploited and provided new insight into disease pathogenesis. However, primary open-angle glaucoma (POAG), known as a complex neurodegenerative disease resulting from retinal ganglion cell death and optic nerve damage, can cause irreversible blindness and visual field loss. POAG, which shares several similarities with Parkinson's disease (PD) and Alzheimer's disease (AD), has limited studies and slow progression in the understanding of pathogenesis when compared to $\mathrm{PD}$ and $\mathrm{AD}$. In this review, we summarized the current knowledge of POAG and commensal microbiota, combined with several lines of evidence in PD and AD to propose a possible hypothesis for POAG pathogenesis: microorganisms cause glaucoma via gut-retina axis, resulting in autoantibodies and autoreactive $\mathrm{T}$ cells that lead to autoimmunity. Furthermore, dual-hit hypothesis, an example of a commensal pathogen that causes PD, was partially exported in POAG. Finally, future perspectives are suggested to expand understanding of POAG.
\end{abstract}

Keywords: primary open-angle glaucoma, microbiota, gut-retina axis, pathogenesis, autoimmunity, neurodegenerative diseases

\section{Introduction}

Primary open-angle glaucoma (POAG) is recognized as an ocular neurodegenerative disease via retinal ganglion cells (RGCs) death and causes irreversible blindness as well as vision defects globally, leading to decreased quality of life [1]. Glaucoma will affect approximately 79.6 million people by 2020 and increase to 111 million by $2040[2,3]$. The disease is classified into

\footnotetext{
*Corresponding author; E-mails: tpteera075@gmail.com; teera.p@ru.ac.th
} 
elevated intraocular pressure (IOP) resulting from abnormal aqueous humor flowing via the development of trabecular meshwork cell fibrosis [4] and normal tension glaucoma (NTG; IOP $<22 \mathrm{mmHg}$ ). The end results of both types are RGCs death via innate and adaptive immunity. In the elevated IOP type, current treatments are IOP reduction composed of surgical intervention or medication. Surgeries may be associated with potential vision-threatening consequences, while the failure rate is approximately $11 \%$ [5-7]. For this reason, medication therapies are considered the primary care option, which is a significant step in minimizing the disease progression in the early stages of glaucomatous eye development. However, currently available pharmacological treatments may cause adverse effects and drug interactions. Long-term treatment can cause subclinical inflammation and conjunctiva fibrosis development leading to increasing failure rates of trabeculectomy [8-11]. The long-term treatment initially requires two or more antiglaucoma drugs, which may predict failure in the present medication $[5,6]$. Importantly, combination drug treatments are widely used for lowering IOP, leading to low patient compliance with multiple daily administrations of eye drops. Consequently, understanding of cellular and molecular pathogenesis is an essential pathway to the development of novel interventions and therapies to overcome glaucoma.

Besides the elevated IOP, NTG patients are also treated with IOP-lowering medication. However, $12 \%$ of patients' treatments are not successful in terms of disease control. Moreover, the "Collaborative Normal-Tension Glaucoma Study" showed that $30 \%$ of IOP reduction was not able to suppress visual loss. These studies indicated that IOP management is not a prominent target for medication [12]. At present, Trivli et al. [13] suggest that this type of glaucoma is closely associated with multifactorial components. The observational epidemiological study shows that the ocular morphology of NTG is associated with visual field defects, suggesting that ocular morphology dysregulation alters the mechanical stress and axonal damage to destroy optic nerve heads and RGCs. However, these findings remain controversial [13, 14]. In addition, vascular disorders composed of vascular dysregulation, neovasculization, and ischemia have been proposed as a pathogenesis of NTG [15]. Gherghel et al. [16] showed that ocular blood flow of a patient, as measured using the mean of a peripheral laser Doppler, was altered depending on the temperature. A cold provocation test illustrates that the instability of both mean ocular perfusion pressure and blood pressure (BP) occurred in patients compared to healthy controls [17]. In a prospective clinical validation study, the NTG patients impaired autonomic cardiovascular regulation, analyzed by an alteration in BP variability and heart rate during resting conditions [18]. In a rabbit experimental model, the instability, induced endothelin-1 injection of the optic nerve, and blood flow resulted in RGC loss [19]. The current model suggests that it is the 
effect of chronic oxidative stress, known as ischemia-reperfusion injury [20]. However, these models do not offer a complete understanding of POAG pathogenesis as there are several factors associated with POAG, such as genetic predisposition and iron-deficiency anemia $[21,22]$. An initiation of POAG is still ambiguous and pathogenesis has not been clearly elucidated.

Previously, the pathophysiological features of glaucoma were compared with other neurodegenerative diseases, especially Parkinson's diseases (PD) and Alzheimer's disease (AD), leading to the reclassification of glaucoma as optic neuropathy with effects in the central nervous system (CNS). The optic nerve is a part of the CNS. Several similarities in clinical manifestations, such as age, presymptomatic stage, clinical progression, genetic predisposition, and cellular pathogenesis, have been found to be shared between glaucoma and neurodegenerative diseases $[23,24]$. For this reason, the study of PD and AD may provide new insight into glaucoma disease.

In the past decade, human microbiota has been rapidly exploited into neurodegenerative diseases and autoimmunity. The alteration of intestinal microbiome into pathogenic bacteria or other groups of bacteria has been broadly recognized as microbial dysbiosis and plays a role as a contributing factor in disease pathogenesis $[25,26]$. Intestinal gut microbiota is well characterized as a confounding factor in several diseases such as amyotrophic lateral sclerosis (ALS), multiple sclerosis (MS), AD, and $\mathrm{PD}$, since the gut microbiome is facile and changed via diet and exercise [27-30]. A microbiota-gut-brain axis is a cross-talk between gut and microbiota, in which enteric bacteria are able to enter the circulation and pass the blood-brain barrier (BBB), known as the humoral pathway, to penetrate the CNS via the vagus nerve, which is a neural pathway $[31,32]$. In addition, a dual-hit hypothesis was applied in PD, where a pathogen invades the brain through an immunoprivileged site like the eye of the host by gut and nose [33]. A population-based follow-up study covering 8 years showed that POAG was significantly associated with $\mathrm{AD}$ and saved as an $\mathrm{AD}$ predictor [34], suggesting that there is a potential link between brain degenerative diseases and glaucoma and retinal degenerative disease. Therefore, this review will summarize and propose the microbiota-gut-retina axis and dual-hit hypothesis as potential pathogenesis in POAG based on current knowledge of brain neurodegenerative diseases and glaucoma studies, which may be applied for the development of a novel therapy.

\section{Commensal Microbiota: From Neurodegenerative Diseases to POAG}

Microbial dysbiosis is a crucial player in the onset of neurological disorders and progressive neuronal loss, including ischemic reperfusion injury [25, 35]. 
In a mouse model, the gut microbiota was strongly associated with AD. For example, intensity-dependent chronic noise exposure reduced gut microbiota diversity and caused the increase of inflammatory mediators, resulting in AD-like effects in the brain [36]. Significant differences in abundance of gut microbiota were shown when comparing between senescence-accelerated mouse prone 8 (SAMP8), recognized as well established deterministic of AD, and senescence-accelerated mouse resistance 1; as such, Lachnospiraceae, Alistipes species, Akkermansia species, and Odoribactor species were enriched in SAMP8 [37]. To confirm this relationship, the Drosophila model with AD pathology showed various groups of commensal bacteria [38]. Recent human study revealed that the bacterial diversity of gut microbiota in AD patients is distinctive from normal controls, such as Actinobacteria, Bacteroides species, Ruminococcus species, Selenomonadales, and Lachnospiraceae [39]. Therefore, these are able to imply that the perturbation of commensal microbiota is involved in the pathogenesis of $\mathrm{AD}$. $\mathrm{Xu}$ and Wang [40] suggested that the gut microbial metabolites should be considered as biomarkers because there is a positive correlation with disease progression in AD.

Regarding AD, several studies of the PD model also found a correlation between gut microbiota and disease pathogenesis, in which dopaminergic neurons were devastated by the aggregation and accumulation of $\alpha$-synuclein $(\alpha \operatorname{Syn})$ $[26,41]$. In dual-hit hypothesis (Braak's hypothesis), the antigens may enter via the nasal and gastric pathways. The enteric nervous system is the early site of $\alpha$ Syn, which subsequently locates in the brain via the vagus nerve $[33,42]$. In the clinical study, the decreasing of Lachnospiraceae and increasing of Lactobacillaceae as well as Christensenellaceae are associated with the severity of idiopathic PD [43]. Furthermore, PD patients were commonly found to have extraordinary increases in coliform bacteria in intestinal microbiome overgrowth [44, 45]. Importantly, there is bias in anti-inflammatory bacteria and pro-inflammatory bacterial microbiome between PD patients and healthy controls. Butyrateproducing bacteria, normally genera Blautia, Coprococcus, and Roseburia, were reduced in the feces of PD. On the contrary, Ralstonia genus, pro-inflammatory bacteria, was predominant in healthy controls compared to PD patients [46]. As a result, the balancing of inflammatory response might regulate gut microbiota, which is associated with PD pathogenesis. A colonic biopsy sample of PD patients, in which dysbiosis was detected by the decreasing of short-chain fatty acids compared to healthy controls, increases Toll-like receptor 4 (TLR4), cytokine expression, and $\mathrm{CD}^{+} \mathrm{T}$ cells. Moreover, TLR4 knockout mouse, induced using oral rotenone, indicated that lower intestinal and motor dysfunction including neurodegenerative and neuroinflammation suggested that innate immunity may play a role in microbial dysbiosis in PD [47]. 
Gastrointestinal permeability saves as an anatomic barrier and one explanation of microbiota and brain damage [25]. Increased permeability is able to disseminate bacterial components or pathogens to the blood and brain [48, 49]. By way of illustration, the level of lipopolysaccharides (LPS) in serum roughly increases in AD and sporadic ALS patients. It is capable of activating monocytes and decreasing IL-10 [50]. In a rat model, the disruption of gut-barrier function and dysbiosis results in bacterial translocation [51]. Importantly, the BBB integrity of $\mathrm{AD}$ is decreased, which leads to immune activation [52]. Another example is a cohort observation in PD patients, where the $\alpha$ Syn, and serum LPS-binding protein, endotoxin marker were increased and associated with intestinal hyperpermeability [53]. Among these studies, it was suggested that gastrointestinal permeability has a connection to the dissemination of antigens or inflammatory factors to the brain.

At present, several diets have the ability to increase epithelial permeability [54] and alter microbiota. As a case in point, unsaturated fatty acid diets affect microbial varieties and increase eightfold in intestinal permeability in a mouse model [55] as well as chicken feeding with different patterns, ad libitum versus restrictive feeding, represented cecal microbiota deviation and may potentially disturb intestinal physiology including morphology and permeability [56]. Furthermore, there is evidence to support that commensal bacteria microorganisms interface with permeability. Chen et al. [57] elucidated that microbial treatment, Lactobacillus rhamnosus GG, improves intestinal permeability and modulates dysbiosis results in a protective role in a sepsis mouse model. Other treatment examples are Puerariae Lobatae Radix and Chuanxiong Rhizoma, which showed that this combination is able to rebalance gut microbiota dysbiosis and revive gut-brain barrier disruption [58].

The roles of commensal microbiota in ocular disease are slowly being explored, but are significantly important [59]. Previously, Horai et al. performed spontaneous uveitis on a mouse model and showed that commensal bacteria activate $\mathrm{T}$ cells by non-cognate interaction and subsequently promote autoreactive $\mathrm{T}$ cells infiltration across blood-retinal barriers. The autoreactive $\mathrm{T}$ cells respond to autoantigen in the retina and result in inflammation [60]. In experimental autoimmune uveitis, the pathology was induced by interphotoreceptor-binding peptides, indicating the alteration of gut microbiome via oral antibiotics administration associated with uveitis severity [61]. A recent study in animal models coupled with adoptive transfer experiments that activated immune response by antigen injection through the anterior chamber, which should activate $\mathrm{T}$ cells in the spleen or thymus via anterior chamber-associated immune deviation (ACAID), showed retinal neurodegeneration resulting from heat shock proteins (HSPs)specific $\mathrm{CD}^{+} \mathrm{Th} 1$, which requires priming from commensal bacteria $[62,63]$. 
Altogether, dysbiosis is also involved in basic glaucoma pathogenesis, similar to other neurodegenerative diseases. Therefore, the microbiota-gut-retinal axis is possible.

A common example of commensal pathogenic bacteria is Helicobacter pylori, which was reclassified as extragastroduodenal diseases such as immune thrombocytopenic purpura and iron-deficiency anemia [64]. Accordingly, H. pylori cause other diseases. Many studies have supported that infected patients significantly increase the risk of developing several neurodegenerative diseases including PD, AD, MS, and POAG [65-68]. In meta-analysis and case-control study, PD patients infected with $H$. pylori showed higher severity of $\mathrm{PD}$ and $H$. pylori eradication therapy to improve disease severity, suggesting that commensal pathogens may contribute to disease deterioration [66]. This is in accordance with glaucoma studies. Kountouras et al. [69] showed that $H$. pylori eradication decreases IOP and improves visual field. In a study by Atilgan et al. [70], the H. pylori infection decreased temporal quadrant retinal nerve fiber layer thickness when compared between pretreatment and posttreatment to eradicate $H$. pylori infection from patients, indicating the early signs of glaucoma. Surprisingly, $H$. pylori can be detected in trabeculectomy specimens of patients, suggesting that bacteria can successfully colonize trabecular meshwork cells [71]. This evidence shows that the microbiota-gut-retina axis is available in our body, although the translocation route remains unknown.

In addition, the specific $H$. pylori IgG antibody is able to be found in the serum and aqueous humor of POAG patients [72, 73]. Increasing the IgG antibody is possible for cross-reactivity with ocular tissue [74]. Previous researches showed that $H$. pylori activate autoantibodies via molecular mimicry in autoimmune disorders such as cardiovascular and autoimmune thyroid disease [75, 76]. H. pylori-seropositive PD patients upregulated eight autoantibodies when compared with $H$. pylori-seronegative PD patients [77]. In glaucomatous tissue, HSPs are increased and play a role in neurodegeneration $[78,79]$, which is a potential target of autoantibodies due to the high level of sequence homology with microbial HSPs [80]. In human studies, HSPsspecific autoantibodies and other autoantibodies increase in the serum and aqueous humor of glaucoma patients [81-83]. Ex vivo stimulation by HSP60 in glaucoma peripheral blood monocyte showed Th2 bias [84]. This study in blood samples of glaucomatous patients compared with non-glaucomatous controls exhibited a trend toward decreased frequency of regulatory T cell. Ex vivo stimulation by a specific antibody to $\varepsilon$ chain of human CD3, CD28, and CD137 revealed $\mathrm{CD}^{+} \mathrm{T}$ response in glaucomatous samples [85]. In an animal model, a glaucomatous formation and RGCs loss were induced by HSPs [86, 87]. Adoptive transfer of $\mathrm{T}$ and $\mathrm{B}$ cells from glaucomatous mice provokes 
detrimental outcome in normal recipient mice [88]. For this reason, it might suggest that $\mathrm{T}$-helper cell and $\mathrm{B}$ cell can circulate to the eye and destroy the retina cells resulting in the progression of visual field loss.

Finally, the other innate immunity may collaborate to augment the pathology of glaucoma. For example, pathogen-associated molecular pattern recognitions are involved in glaucoma pathogenesis. For instance, the TLR4 may be the critical player in recognizing HSPs as damage-associated molecular patterns in glaucoma [89]. This is consistent with $H$. pylori, producing miscellaneous antigens that activate the immune system by TLRs and increase TLR4 expression in gastric epithelial cells [90, 91]. Taken together, a commensal bacterium such as $H$. pylori may be one actor in glaucoma pathogenesis by causing autoimmunity.

In summary, we proposed a hypothesis for glaucoma, neurodegenerative disease, and autoimmunity resulting from orchestrations of $\mathrm{T}$ and $\mathrm{B}$ cells, in which bacteria or endotoxins are able to migrate to the ocular region using the microbiota-gut-retina axis via alteration of gastrointestinal permeability and increased inflammatory cytokines and autoantibodies in the blood and aqueous humor. H. pylori are able to enter the eye through unknown routes of translocation. Commensal bacteria can shed antigens to blood and is subsequently captured in the spleen, an important organ of ocular immunity, which causes ACAID, resulting in immune cell activation as well as homing to the ocular region. Furthermore, the antigens may appear in the anterior chamber resulting in Th1 development, which requires the commensal gut microbiota. Therefore, dysbiosis may initiate the step of POAG development by priming immunity (Figure 1).

\section{Oral Microbiota: A Possible Source of Antigens That May Cause Glaucoma}

Other examples of microbiota in neurodegenerative diseases are oral microbiota dysbiosis. Oral pathogens such as Porphyromonas gingivalis and Treponema forsythia are associated with $\mathrm{AD}$ and able to be found in the brain of AD patients [92-94]. Recently, the reduction of $P$. gingivalis toxin, gingipains, was able to decrease the bacterial load in the brain and abate neuroinflammation [95]. However, no direct evidence has shown that oral pathogens are associated with glaucoma. In this field, Astafur et al. carried out the first study that showed oral bacteria load significantly increases in mouthwash specimens from glaucoma patients using 16s RNA and pyrosequencing. However, there are confounding factors appearing in this study. The results showed significant differences in age, gender, and diabetes status [96]. Consequently, the data were stratified and showed 


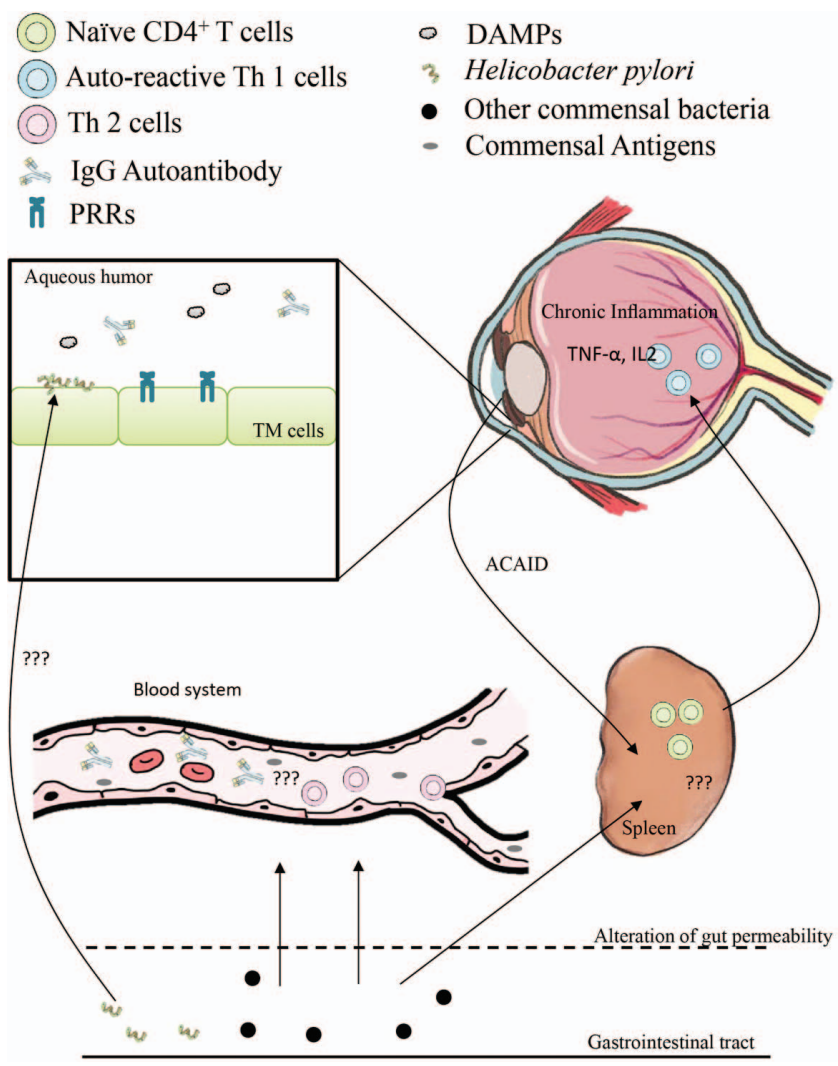

Figure 1. The possible model of glaucoma pathogenesis is autoimmunity disease. The gut microbiota is able to shed the antigens or translocate the bacterium to the outside of the gastrointestinal tract via the alteration of epithelial permeability. The antigens may activate Th2 development and increase antibodies by unknown mechanisms. Moreover, the routes of bacteria migration are undercharacterized. The trabecular meshwork (TM) cells are able to recognize both autoantigens, the bacteria antigens, and damage-associated molecular pattern (DAMPs) using pathogen-associated molecular pattern (PAMPs), such as TLR4, and autoantibodies to generate imbalanced signaling of inflammation, which causes retinal damage as a consequence. In addition, the activation of autoreactive $\mathrm{T}$ cells by antigen induction in anterior chamber results in anterior chamber-associated immune deviation (ACAID). This mechanism is not fully understood, although it requires the commensal microbiota, resulting in Th1 development and inflammation in the eye.

This should be further investigated in the future

Streptococcus spp. in glaucoma cases when compared with control [97]. Recently, dental health identified by the number of natural teeth was proposed as a marker for glaucoma. However, there are some controversies that should be addressed and validated in a large sample group. Moreover, classified periodontal status with other factors may affect the study [98, 99]. 


\section{Nutrition Behavior May be Considered as Supplement for Glaucoma Patients}

As previously mentioned, gastrointestinal permeability is altered depending on diet and microbiota. Supplementation of high fat and protein diets was an increase of bacteria in the gut such as Alistipes, Bacteroides, and Bilophila organisms, whereas the diet enriched with a high sugar caused a decrease of the beneficial gut microbiome (Lactobacillus, Ruminococcaceae, and Lachnospiraceaeae) [100-102]. These may directly or indirectly affect the cause of glaucoma. Several lines of evidence suggested that omega fatty acids, caffeine, and ketogenic diet have been reported to provide a neuroprotective role in glaucoma [103, 104]. In the clinical study, carbohydrate ingestion in POAG patients showed systemic autonomic dysregulation [105]. Ketogenic diets, modified gut microbiota, and permeability may play a role in the neuroprotection of glaucoma [106-108]. A population-based study in Japan suggested the consumption of meat, which is positively associated with open-angle glaucoma [109]. In Canada, a multicenter cross-sectional study reported that approximately one in nine glaucoma patients apply complementary and alternative medicine, including diets, for their disease [110]. Accumulation of studies showed that these diets convert gut microbiome [107].

Nowadays, the current research suggested that an individual's diet might have an impact on IOP and progression of the disease [111]. It may help people to improve and maintain their eyesight. A study found that salt-enriched diet intake is associated with a decreasing IOP in the eyes [112]. Thus, proper salt consumption may be the benefit for glaucoma patients. In addition, it is also found that combination of retinol and vitamin B1 seems to involve in glaucoma progression especially in the high dose [113]. Moreover, high-antioxidant foods, including leafy green vegetables, flavonoid-rich fruits, and red wine, propose a low risk of glaucoma [114-116]. In this regard, we speculated that there is some relation between diets, commensal microbiota, and immunity in the eye, which may cause inflammation and retina damage, resulting in glaucoma. Therefore, we suggested that to improve the effectiveness of lowering IOP medication, nutrition management should not be neglected.

\section{Further Perspective}

POAG is a disease that significantly affects the quality of life. Current medication therapies are able to slow disease progressions but may be associated with drug resistance during prolonged treatment. Therefore, understanding of glaucoma pathogenesis is a significantly pivotal approach. At present, innate 
lymphoid cells play a key role in autoimmunity, although glaucoma has a gap in this area. Moreover, the microbiome has rapidly progressed. The gut-retina axis is possible, as mentioned earlier. The route of migration should be identified in the future. How the gut-microbiota activates $\mathrm{T}$ and $\mathrm{B}$ cells to produce the autoantigens, which are able to react with ocular antigens? How many cell types are associated with this mechanism? In addition, how the ACAID involved in glaucoma and autoimmunity should be linked with gut microbiota? These uninvestigated questions were shown in Figure 1. Finally, oral microbiota is also important due to the association between oral infection and neurodegenerative diseases. To answer this question, a cohort study of periodontal disease and commensal infection should not be neglected in glaucoma study. Finally, diet may be adopted as a contributing factor for glaucoma pathogenesis and may be applied in combinatorial treatment for glaucoma patients. These aspects should be confirmed in a laboratory with epidemiological effort.

\section{Conclusions}

POAG concepts have been modified in the past decade. Advances in science and technology have been exploring and explaining glaucoma pathogenesis from several aspects, especially autoimmunity. Our review summarized the relationship between glaucoma and commensal bacteria, which resulted in one possible hypothesis: microorganisms prime the immune cells to breakdown self-tolerance and cause autoimmunity. The commensal dysbiosis locates to the eye by gut-retina axis, although the route of translocation is underinvestigated. For this reason, a sufficient-component cause model should be revised or reconsidered. Finally, we suggested a potential way to slow the progression of the disease using diet coupled with medication treatment. However, the pathogenesis has multidisciplinary factors that affect and cause the disease.

\section{Acknowledgements}

Dr. TP and NC are grateful for the support provided by the Faculty of Optometry, Ramkhamhaeng University, Thailand. This review was not a part of the project, which obtained a funding from the university or other companies. This article did not receive any specific grant from funding agencies in the public, commercial, or not-for-profit sectors. Therefore, the funding source had no such involvement in the body and detail of the article. 


\section{Conflict of Interest}

The authors declare no conflict of interest regarding the publication of this paper.

\section{References}

1. Chun, Y. S., Sung, K. R., Park, C. K., Kim, H. K., Yoo, C., Kim, Y. Y., Park, K. H., Kim, C. Y., Choi, K. R., Lee, K. W., Han, S., Kim, C. S., LIGHT (Life Quality of Glaucoma Patients Who Underwent Treatment) Study of the Korean Glaucoma Society: Vision-related quality of life according to location of visual field loss in patients with glaucoma. Acta Ophthalmol 97, e772-e779 (2019).

2. Tham, Y. C., Li, X., Wong, T. Y., Quigley, H. A., Aung, T., Cheng, C. Y.: Global prevalence of glaucoma and projections of glaucoma burden through 2040: A systematic review and meta-analysis. Ophthalmology 121, 2081-2090 (2014).

3. Quigley, H. A., Broman, A. T.: The number of people with glaucoma worldwide in 2010 and 2020. Br J Ophthalmol 90, 262-267 (2006).

4. Wei, X., Cho, K. S., Thee, E. F., Jager, M. J., Chen, D. F.: Neuroinflammation and microglia in glaucoma: Time for a paradigm shift. J Neurosci Res 97, 70-76 (2019).

5. Lu, L. J., Tsai, J. C., Liu, J.: Novel pharmacologic candidates for treatment of primary open-angle glaucoma. Yale J Biol Med 90, 111-118 (2017).

6. Motlagh, B. F.: Medical therapy versus trabeculectomy in patients with open-angle glaucoma. Arq Bras Oftalmol 79, 233-237 (2016).

7. Burr, J., Azuara-Blanco, A., Avenell, A., Tuulonen, A.: Medical versus surgical interventions for open angle glaucoma. Cochrane Database Syst Rev 9, CD004399 (2012).

8. Skuta, G. L., Parrish, R. K., 2nd: Wound healing in glaucoma filtering surgery. Surv Ophthalmol 32, 149-170 (1987).

9. Schwab, I. R., Linberg, J. V., Gioia, V. M., Benson, W. H., Chao, G. M.: Foreshortening of the inferior conjunctival fornix associated with chronic glaucoma medications. Ophthalmology 99, 197-202 (1992).

10. Broadway, D. C., Grierson, I., O’Brien, C., Hitchings, R. A.: Adverse effects of topical antiglaucoma medication. I. The conjunctival cell profile. Arch Ophthalmol 112, 1437-1445 (1994).

11. Broadway, D. C., Grierson, I., O’Brien, C., Hitchings, R. A.: Adverse effects of topical antiglaucoma medication. II. The outcome of filtration surgery. Arch Ophthalmol 112, 1446-1454 (1994).

12. Collaborative Normal-Tension Glaucoma Study Group: The effectiveness of intraocular pressure reduction in the treatment of normal-tension glaucoma. Collaborative NormalTension Glaucoma Study Group. Am J Ophthalmol 126, 498-505 (1998).

13. Trivli, A., Koliarakis, I., Terzidou, C., Goulielmos, G. N., Siganos, C. S., Spandidos, D. A., Dalianis, G., Detorakis, E. T.: Normal-tension glaucoma: Pathogenesis and genetics. Exp Ther Med 17, 563-574 (2019).

14. Lee, J. W., Wong, R. L., Chan, J. C., Wong, I. Y., Lai, J. S.: Differences in corneal parameters between normal tension glaucoma and primary open-angle glaucoma. Int Ophthalmol 35, 67-72 (2015). 
15. Xu, H., Zhai, R., Zong, Y., Kong, X., Jiang, C., Sun, X., He, Y., Li, X.: Comparison of retinal microvascular changes in eyes with high-tension glaucoma or normal-tension glaucoma: A quantitative optic coherence tomography angiographic study. Graefes Arch Clin Exp Ophthalmol 256, 1179-1186 (2018).

16. Gherghel, D., Hosking, S. L., Cunliffe, I. A.: Abnormal systemic and ocular vascular response to temperature provocation in primary open-angle glaucoma patients: A case for autonomic failure? Invest Ophthalmol Vis Sci 45, 3546-3554 (2004).

17. Kurysheva, N. I., Ryabova, T. Y., Shlapak, V. N.: Heart rate variability: The comparison between high tension and normal tension glaucoma. EPMA J 9, 35-45 (2018).

18. Lindemann, F., Kuerten, D., Koch, E., Fuest, M., Fischer, C., Voss, A., Plange, N.: Blood pressure and heart rate variability in primary open-angle glaucoma and normal tension glaucoma. Curr Eye Res 43, 1507-1513 (2018).

19. Cioffi, G. A., Sullivan, P.: The effect of chronic ischemia on the primate optic nerve. Eur J Ophthalmol 9, S34-S36 (1999).

20. Mozaffarieh, M., Flammer, J.: New insights in the pathogenesis and treatment of normal tension glaucoma. Curr Opin Pharmacol 13, 43-49 (2013).

21. Firat, P. G., Demirel, E. E., Dikci, S., Kuku, I., Genc, O.: Evaluation of iron deficiency anemia frequency as a risk factor in glaucoma. Anemia 2018, 1456323 (2018).

22. Chen, Y. T., Chen, S. N., Liu, C. S.: The relationship between optic atrophy 1 polymorphism and normal tension glaucoma in Taiwan. Taiwan J Ophthalmol 8, 82-86 (2018).

23. Danesh-Meyer, H. V., Levin, L. A.: Glaucoma as a neurodegenerative disease. J Neuroophthalmol 35, S22-S28 (2015).

24. Gupta, N., Yucel, Y. H.: Glaucoma as a neurodegenerative disease. Curr Opin Ophthalmol 18, 110-114 (2007).

25. Spielman, L. J., Gibson, D. L., Klegeris, A.: Unhealthy gut, unhealthy brain: The role of the intestinal microbiota in neurodegenerative diseases. Neurochem Int 120, 149-163 (2018).

26. Dehhaghi, M., Kazemi Shariat Panahi, H., Guillemin, G. J.: Microorganisms' footprint in neurodegenerative diseases. Front Cell Neurosci 12, 466 (2018).

27. Baxter, N. T., Schmidt, A. W., Venkataraman, A., Kim, K. S., Waldron, C., Schmidt, T. M.: Dynamics of human gut microbiota and short-chain fatty acids in response to dietary interventions with three fermentable fibers. MBio 10, e02566-18 (2019).

28. Thirumangalakudi, L., Prakasam, A., Zhang, R., Bimonte-Nelson, H., Sambamurti, K., Kindy, M. S., Bhat, N. R.: High cholesterol-induced neuroinflammation and amyloid precursor protein processing correlate with loss of working memory in mice. J Neurochem 106, 475-485 (2008).

29. Agarwal, P., Wang, Y., Buchman, A. S., Holland, T. M., Bennett, D. A., Morris, M. C.: MIND diet associated with reduced incidence and delayed progression of Parkinsonism A in old age. J Nutr Health Aging 22, 1211-1215 (2018).

30. Wlodarek, D.: Role of ketogenic diets in neurodegenerative diseases (Alzheimer's disease and Parkinson's disease). Nutrients 11, 169 (2019).

31. Sandhu, K. V., Sherwin, E., Schellekens, H., Stanton, C., Dinan, T. G., Cryan, J. F.: Feeding the microbiota-gut-brain axis: Diet, microbiome, and neuropsychiatry. Transl Res 179, 223-244 (2017). 
32. Dumitrescu, L., Popescu-Olaru, I., Cozma, L., Tulba, D., Hinescu, M. E., Ceafalan, L. C., Gherghiceanu, M., Popescu, B. O.: Oxidative stress and the microbiota-gut-brain axis. Oxid Med Cell Longev 2018, 2406594 (2018).

33. Hawkes, C. H., Del Tredici, K., Braak, H.: Parkinson's disease: A dual-hit hypothesis. Neuropathol Appl Neurobiol 33, 599-614 (2007).

34. Lin, I. C., Wang, Y. H., Wang, T. J., Wang, I. J., Shen, Y. D., Chi, N. F., Chien, L. N.: Glaucoma, Alzheimer's disease, and Parkinson's disease: An 8-year population-based follow-up study. PLoS One 9, e108938 (2014).

35. Giau, V. V., Wu, S. Y., Jamerlan, A., An, S. S. A., Kim, S. Y., Hulme, J.: Gut microbiota and their neuroinflammatory implications in Alzheimer's disease. Nutrients 10, E1765 (2018).

36. Cui, B., Su, D., Li, W., She, X., Zhang, M., Wang, R., Zhai, Q.: Effects of chronic noise exposure on the microbiome-gut-brain axis in senescence-accelerated prone mice: Implications for Alzheimer's disease. J Neuroinflammation 15, 190 (2018).

37. Peng, W., Yi, P., Yang, J., Xu, P., Wang, Y., Zhang, Z., Huang, S., Wang, Z., Zhang, C.: Association of gut microbiota composition and function with a senescence-accelerated mouse model of Alzheimer's disease using 16S rRNA gene and metagenomic sequencing analysis. Aging (Albany NY) 10, 4054-4065 (2018).

38. Kong, Y., Jiang, B., Luo, X.: Gut microbiota influences Alzheimer's disease pathogenesis by regulating acetate in Drosophila model. Future Microbiol 13, 1117-1128 (2018).

39. Zhuang, Z. Q., Shen, L. L., Li, W. W., Fu, X., Zeng, F., Gui, L., Lu, Y., Cai, M., Zhu, C., Tan, Y. L., Zheng, P., Li, H. Y., Zhu, J., Zhou, H. D., Bu, X. L., Wang, Y. J.: Gut microbiota is altered in patients with Alzheimer's disease. J Alzheimers Dis 63, 1337-1346 (2018).

40. Xu, R., Wang, Q.: Towards understanding brain-gut-microbiome connections in Alzheimer's disease. BMC Syst Biol 10, 63 (2016).

41. Brettschneider, J., Del Tredici, K., Lee, V. M., Trojanowski, J. Q.: Spreading of pathology in neurodegenerative diseases: A focus on human studies. Nat Rev Neurosci 16, 109-120 (2015).

42. Del Tredici, K., Braak, H.: A not entirely benign procedure: Progression of Parkinson's disease. Acta Neuropathol 115, 379-384 (2008).

43. Barichella, M., Severgnini, M., Cilia, R., Cassani, E., Bolliri, C., Caronni, S., Ferri, V., Cancello, R., Ceccarani, C., Faierman, S., Pinelli, G., De Bellis, G., Zecca, L., Cereda, E., Consolandi, C., Pezzoli, G.: Unraveling gut microbiota in Parkinson's disease and atypical parkinsonism. Mov Disord 34(3), 396-405 (2018).

44. Tan, A. H., Mahadeva, S., Thalha, A. M., Gibson, P. R., Kiew, C. K., Yeat, C. M., Ng, S. W., Ang, S. P., Chow, S. K., Tan, C. T., Yong, H. S., Marras, C., Fox, S. H., Lim, S. Y.: Small intestinal bacterial overgrowth in Parkinson's disease. Parkinsonism Relat Disord 20, 535-540 (2014).

45. Gabrielli, M., Bonazzi, P., Scarpellini, E., Bendia, E., Lauritano, E. C., Fasano, A., Ceravolo, M. G., Capecci, M., Rita Bentivoglio, A., Provinciali, L., Tonali, P. A., Gasbarrini, A.: Prevalence of small intestinal bacterial overgrowth in Parkinson's disease. Mov Disord 26, 889-892 (2011).

46. Keshavarzian, A., Green, S. J., Engen, P. A., Voigt, R. M., Naqib, A., Forsyth, C. B., Mutlu, E., Shannon, K. M.: Colonic bacterial composition in Parkinson's disease. Mov Disord 30, 1351-1360 (2015). 
47. Perez-Pardo, P., Dodiya, H. B., Engen, P. A., Forsyth, C. B., Huschens, A. M., Shaikh, M., Voigt, R. M., Naqib, A., Green, S. J., Kordower, J. H., Shannon, K. M., Garssen, J., Kraneveld, A. D., Keshavarzian, A.: Role of TLR4 in the gut-brain axis in Parkinson's disease: A translational study from men to mice. Gut 68, 829-843 (2018).

48. Obrenovich, M. E. M.: Leaky gut, leaky brain? Microorganisms 6, 107 (2018).

49. Yacyshyn, B., Meddings, J., Sadowski, D., Bowen-Yacyshyn, M. B.: Multiple sclerosis patients have peripheral blood CD45RO+ B cells and increased intestinal permeability. Dig Dis Sci 41, 2493-2498 (1996).

50. Zhang, R., Miller, R. G., Gascon, R., Champion, S., Katz, J., Lancero, M., Narvaez, A., Honrada, R., Ruvalcaba, D., McGrath, M. S.: Circulating endotoxin and systemic immune activation in sporadic amyotrophic lateral sclerosis (sALS). J Neuroimmunol 206, 121-124 (2009).

51. Munoz, L., Borrero, M. J., Ubeda, M., Conde, E., Del Campo, R., Rodriguez-Serrano, M., Lario, M., Sanchez-Diaz, A. M., Pastor, O., Diaz, D., Garcia-Bermejo, L., Monserrat, J., Alvarez-Mon, M., Albillos, A.: Intestinal immune dysregulation driven by dysbiosis promotes barrier disruption and bacterial translocation in rats with cirrhosis. Hepatology 70, 925-938 (2019).

52. Wada, H.: Blood-brain barrier permeability of the demented elderly as studied by cerebrospinal fluid-serum albumin ratio. Intern Med 37, 509-513 (1998).

53. Berdel, W. E., Okamoto, S.: Ether lipids in cancer chemotherapy. Keio J Med 39, 75-78 (1990).

54. Shen, Q. X., Xu, G. X., Shen, M. H.: Effect of early enteral nutrition (EN) on endotoxin in serum and intestinal permeability in patients with severe acute pancreatitis. Eur Rev Med Pharmacol Sci 21, 2764-2768 (2017).

55. Cani, P. D., Bibiloni, R., Knauf, C., Waget, A., Neyrinck, A. M., Delzenne, N. M., Burcelin, R.: Changes in gut microbiota control metabolic endotoxemia-induced inflammation in high-fat diet-induced obesity and diabetes in mice. Diabetes 57, 1470-1481 (2008).

56. Metzler-Zebeli, B. U., Siegerstetter, S. C., Magowan, E., Lawlor, P. G., Petri, R. M., Ne, O. C., Zebeli, Q.: Feed restriction modifies intestinal microbiota-host mucosal networking in chickens divergent in residual feed intake. mSystems 4 (2019).

57. Chen, L., Li, H., Li, J., Chen, Y., Yang, Y.: Lactobacillus rhamnosus GG treatment improves intestinal permeability and modulates microbiota dysbiosis in an experimental model of sepsis. Int J Mol Med 43, 1139-1148 (2019).

58. Chen, R., Wu, P., Cai, Z., Fang, Y., Zhou, H., Lasanajak, Y., Tang, L., Ye, L., Hou, C., Zhao, J.: Puerariae Lobatae Radix with chuanxiong Rhizoma for treatment of cerebral ischemic stroke by remodeling gut microbiota to regulate the brain-gut barriers of dietary capsaicin against chronic low-grade inflammation. J Nutr Biochem 65, 101-114 (2018).

59. Wen, X., Hu, X., Miao, L., Ge, X., Deng, Y., Bible, P. W., Wei, L.: Epigenetics, microbiota, and intraocular inflammation: New paradigms of immune regulation in the eye. Prog Retin Eye Res 64, 84-95 (2018).

60. Horai, R., Zarate-Blades, C. R., Dillenburg-Pilla, P., Chen, J., Kielczewski, J. L., Silver, P. B., Jittayasothorn, Y., Chan, C. C., Yamane, H., Honda, K., Caspi, R. R.: Microbiotadependent activation of an autoreactive $\mathrm{T}$ cell receptor provokes autoimmunity in an immunologically privileged site. Immunity 43, 343-353 (2015). 
61. Nakamura, Y. K., Metea, C., Karstens, L., Asquith, M., Gruner, H., Moscibrocki, C., Lee, I., Brislawn, C. J., Jansson, J. K., Rosenbaum, J. T., Lin, P.: Gut microbial alterations associated with protection from autoimmune Uveitis. Invest Ophthalmol Vis Sci 57, 3747-3758 (2016).

62. Chen, H., Cho, K. S., Vu, T. H. K., Shen, C. H., Kaur, M., Chen, G., Mathew, R., McHam, M. L., Fazelat, A., Lashkari, K., Au, N. P. B., Tse, J. K. Y., Li, Y., Yu, H., Yang, L., SteinStreilein, J., Ma, C. H. E., Woolf, C. J., Whary, M. T., Jager, M. J., Fox, J. G., Chen, J., Chen, D. F.: Commensal microflora-induced $\mathrm{T}$ cell responses mediate progressive neurodegeneration in glaucoma. Nat Commun 9, 3209 (2018).

63. Benhar, I., London, A., Schwartz, M.: The privileged immunity of immune privileged organs: The case of the eye. Front Immunol 3, 296 (2012).

64. Tsay, F. W., Hsu, P. I.: H. pylori infection and extra-gastroduodenal diseases. J Biomed Sci 25, 65 (2018).

65. Jaruvongvanich, V., Sanguankeo, A., Jaruvongvanich, S., Upala, S.: Association between Helicobacter pylori infection and multiple sclerosis: A systematic review and meta-analysis. Mult Scler Relat Disord 7, 92-97 (2016).

66. Dardiotis, E., Tsouris, Z., Mentis, A. A., Siokas, V., Michalopoulou, A., Sokratous, M., Dastamani, M., Bogdanos, D. P., Deretzi, G., Kountouras, J.: H. pylori and Parkinson's disease: Meta-analyses including clinical severity. Clin Neurol Neurosurg 175, 16-24 (2018).

67. Fani, L., Wolters, F. J., Ikram, M. K., Bruno, M. J., Hofman, A., Koudstaal, P. J., Darwish Murad, S., Ikram, M. A.: Helicobacter pylori and the risk of dementia: A population-based study. Alzheimers Dement 14, 1377-1382 (2018).

68. Zeng, J., Liu, H., Liu, X., Ding, C.: The relationship between Helicobacter pylori infection and open-angle glaucoma: A meta-analysis. Invest Ophthalmol Vis Sci 56, 5238-5245 (2015).

69. Kountouras, J., Mylopoulos, N., Chatzopoulos, D., Zavos, C., Boura, P., Konstas, A. G., Venizelos, J.: Eradication of Helicobacter pylori may be beneficial in the management of chronic open-angle glaucoma. Arch Intern Med 162, 1237-1244 (2002).

70. Atilgan, C. U., Kosekahya, P., Yozgat, A., Sen, E., Berker, N., Caglayan, M., Sendul, S. Y., Altiparmak, E., Yilmazbas, P.: Are optic nerve heads of patients with Helicobacter pylori infection more susceptible to glaucomatous damage? Helicobacter 22, 1-6 (2017).

71. Zavos, C., Kountouras, J., Sakkias, G., Venizelos, I., Deretzi, G., Arapoglou, S.: Histological presence of Helicobacter pylori bacteria in the trabeculum and iris of patients with primary open-angle glaucoma. Ophthalmic Res 47, 150-156 (2012).

72. Kountouras, J., Mylopoulos, N., Boura, P., Bessas, C., Chatzopoulos, D., Venizelos, J., Zavos, C.: Relationship between Helicobacter pylori infection and glaucoma. Ophthalmology 108, 599-604 (2001).

73. Deshpande, N., Lalitha, P., Krishna das, S. R., Jethani, J., Pillai, R. M., Robin, A., Karthik: Helicobacter pylori $\operatorname{IgG}$ antibodies in aqueous humor and serum of subjects with primary open angle and pseudo-exfoliation glaucoma in a South Indian population. J Glaucoma 17, 605-610 (2008).

74. Kountouras, J., Mylopoulos, N., Konstas, A. G., Zavos, C., Chatzopoulos, D., Boukla, A.: Increased levels of Helicobacter pylori IgG antibodies in aqueous humor of patients with primary open-angle and exfoliation glaucoma. Graefes Arch Clin Exp Ophthalmol 241, 884-890 (2003). 
75. Chmiela, M., Gonciarz, W.: Molecular mimicry in Helicobacter pylori infections. World J Gastroenterol 23, 3964-3977 (2017).

76. Choi, Y. M., Kim, T. Y., Kim, E. Y., Jang, E. K., Jeon, M. J., Kim, W. G., Shong, Y. K., Kim, W. B.: Association between thyroid autoimmunity and Helicobacter pylori infection. Korean J Intern Med 32, 309-313 (2017).

77. Suwarnalata, G., Tan, A. H., Isa, H., Gudimella, R., Anwar, A., Loke, M. F., Mahadeva, S., Lim, S. Y., Vadivelu, J.: Augmentation of autoantibodies by Helicobacter pylori in Parkinson's disease patients may be linked to greater severity. PLoS One 11, e0153725 (2016).

78. Chen, S., Brown, I. R.: Neuronal expression of constitutive heat shock proteins: Implications for neurodegenerative diseases. Cell Stress Chaperones 12, 51-58 (2007).

79. Cao, Y., Gao, L., Tang, R., Zhang, W.: Hsp70 protects human trabecular meshwork cells injury induced by UVB through Smad pathway. Pharmazie 72, 334-337 (2017).

80. Magen, E., Delgado, J. S.: Helicobacter pylori and skin autoimmune diseases. World J Gastroenterol 20, 1510-1516 (2014).

81. Tezel, G., Seigel, G. M., Wax, M. B.: Autoantibodies to small heat shock proteins in glaucoma. Invest Ophthalmol Vis Sci 39, 2277-2287 (1998).

82. Wax, M. B., Tezel, G., Kawase, K., Kitazawa, Y.: Serum autoantibodies to heat shock proteins in glaucoma patients from Japan and the United States. Ophthalmology 108, 296-302 (2001).

83. Joachim, S. C., Bruns, K., Lackner, K. J., Pfeiffer, N., Grus, F. H.: Antibodies to alpha B-crystallin, vimentin, and heat shock protein 70 in aqueous humor of patients with normal tension glaucoma and IgG antibody patterns against retinal antigen in aqueous humor. Curr Eye Res 32, 501-509 (2007).

84. Guo, C., Wu, N., Niu, X., Wu, Y., Chen, D., Guo, W.: Comparison of T helper cell patterns in primary open-angle glaucoma and normal-pressure glaucoma. Med Sci Monit 24, 1988-1996 (2018).

85. Yang, X., Zeng, Q., Goktas, E., Gopal, K., Al-Aswad, L., Blumberg, D. M., Cioffi, G. A., Liebmann, J. M., Tezel, G.: T-lymphocyte subset distribution and activity in patients with glaucoma. Invest Ophthalmol Vis Sci 60, 877-888 (2019).

86. Wax, M. B., Tezel, G., Yang, J., Peng, G., Patil, R. V., Agarwal, N., Sappington, R. M., Calkins, D. J.: Induced autoimmunity to heat shock proteins elicits glaucomatous loss of retinal ganglion cell neurons via activated T-cell-derived fas-ligand. J Neurosci 28 , 12085-12096 (2008).

87. Joachim, S. C., Wax, M. B., Seidel, P., Pfeiffer, N., Grus, F. H.: Enhanced characterization of serum autoantibody reactivity following HSP 60 immunization in a rat model of experimental autoimmune glaucoma. Curr Eye Res 35, 900-908 (2010).

88. Gramlich, O. W., Ding, Q. J., Zhu, W., Cook, A., Anderson, M. G., Kuehn, M. H.: Adoptive transfer of immune cells from glaucomatous mice provokes retinal ganglion cell loss in recipients. Acta Neuropathol Commun 3, 56 (2015).

89. Poyomtip, T.: Roles of toll-like receptor 4 for cellular pathogenesis in primary open-angle glaucoma: A potential therapeutic strategy. J Microbiol Immunol Infect 52, 201-206 (2018).

90. Pachathundikandi, S. K., Lind, J., Tegtmeyer, N., El-Omar, E. M., Backert, S.: Interplay of the gastric pathogen Helicobacter pylori with toll-like receptors. Biomed Res Int 2015, 192420 (2015). 
91. Su, B., Ceponis, P. J., Lebel, S., Huynh, H., Sherman, P. M.: Helicobacter pylori activates Toll-like receptor 4 expression in gastrointestinal epithelial cells. Infect Immun 71, 3496-3502 (2003).

92. Bell, J. S., Spencer, J. I., Yates, R. L., Yee, S. A., Jacobs, B. M., DeLuca, G. C.: Invited review: From nose to gut - The role of the microbiome in neurological disease. Neuropathol Appl Neurobiol 45, 195-215 (2019).

93. Aguayo, S., Schuh, C., Vicente, B., Aguayo, L. G.: Association between Alzheimer's disease and oral and gut microbiota: Are pore forming proteins the missing link? J Alzheimers Dis 65, 29-46 (2018).

94. Ranjan, R., Abhinay, A., Mishra, M.: Can oral microbial infections be a risk factor for neurodegeneration? A review of the literature. Neurol India 66, 344-351 (2018).

95. Dominy, S. S., Lynch, C., Ermini, F., Benedyk, M., Marczyk, A., Konradi, A., Nguyen, M., Haditsch, U., Raha, D., Griffin, C., Holsinger, L. J., Arastu-Kapur, S., Kaba, S., Lee, A., Ryder, M. I., Potempa, B., Mydel, P., Hellvard, A., Adamowicz, K., Hasturk, H., Walker, G. D., Reynolds, E. C., Faull, R. L. M., Curtis, M. A., Dragunow, M., Potempa, J.: Porphyromonas gingivalis in Alzheimer's disease brains: Evidence for disease causation and treatment with small-molecule inhibitors. Sci Adv 5, eaau3333 (2019).

96. Astafurov, K., Elhawy, E., Ren, L., Dong, C. Q., Igboin, C., Hyman, L., Griffen, A., Mittag, T., Danias, J.: Oral microbiome link to neurodegeneration in glaucoma. PLoS One 9, e104416 (2014).

97. Polla, D., Astafurov, K., Hawy, E., Hyman, L., Hou, W., Danias, J.: A pilot study to evaluate the oral microbiome and dental health in primary open-angle glaucoma. J Glaucoma 26, 320-327 (2017).

98. Agrawal, K., Agrawal, R.: Re: Pasquale et al.: Prospective study of oral health and risk of primary open-angle glaucoma in men: Data from the health professionals follow-up study (Ophthalmology. 2016;123:2318-2327). Ophthalmology 124, e49-e50 (2017).

99. Pasquale, L. R., Hyman, L., Wiggs, J. L., Rosner, B. A., Joshipura, K., McEvoy, M., McPherson, Z. E., Danias, J., Kang, J. H.: Prospective study of oral health and risk of primary open-angle glaucoma in men: Data from the health professionals follow-up study. Ophthalmology 123, 2318-2327 (2016).

100. David, L. A., Maurice, C. F., Carmody, R. N., Gootenberg, D. B., Button, J. E., Wolfe, B. E., Ling, A. V., Devlin, A. S., Varma, Y., Fischbach, M. A., Biddinger, S. B., Dutton, R. J., Turnbaugh, P. J.: Diet rapidly and reproducibly alters the human gut microbiome. Nature 505, 559-563 (2014).

101. Beilharz, J. E., Kaakoush, N. O., Maniam, J., Morris, M. J.: The effect of short-term exposure to energy-matched diets enriched in fat or sugar on memory, gut microbiota and markers of brain inflammation and plasticity. Brain Behav Immun 57, 304-313 (2016).

102. Daniel, H., Gholami, A. M., Berry, D., Desmarchelier, C., Hahne, H., Loh, G., Mondot, S., Lepage, P., Rothballer, M., Walker, A., Bohm, C., Wenning, M., Wagner, M., Blaut, M., Schmitt-Kopplin, P., Kuster, B., Haller, D., Clavel, T.: High-fat diet alters gut microbiota physiology in mice. ISME J 8, 295-308 (2014).

103. Zarnowski, T., Tulidowicz-Bielak, M., Zarnowska, I., Mitosek-Szewczyk, K., Wnorowski, A., Jozwiak, K., Gasior, M., Turski, W. A.: Kynurenic acid and neuroprotective activity of the ketogenic diet in the eye. Curr Med Chem 24, 3547-3558 (2017).

104. Perez, C. I., Singh, K., Lin, S.: Relationship of lifestyle, exercise, and nutrition with glaucoma. Curr Opin Ophthalmol 30, 82-88 (2019). 
105. Cao, L., Graham, S. L., Pilowsky, P. M.: Carbohydrate ingestion induces differential autonomic dysregulation in normal-tension glaucoma and primary open angle glaucoma. PLoS One 13, e0198432 (2018).

106. Ma, D., Wang, A. C., Parikh, I., Green, S. J., Hoffman, J. D., Chlipala, G., Murphy, M. P., Sokola, B. S., Bauer, B., Hartz, A. M. S., Lin, A. L.: Ketogenic diet enhances neurovascular function with altered gut microbiome in young healthy mice. Sci Rep 8, 6670 (2018).

107. Newell, C., Bomhof, M. R., Reimer, R. A., Hittel, D. S., Rho, J. M., Shearer, J.: Ketogenic diet modifies the gut microbiota in a murine model of autism spectrum disorder. Mol Autism 7, 37 (2016).

108. Zarnowski, T., Tulidowicz-Bielak, M., Kosior-Jarecka, E., Zarnowska, I., Turski, W. A., Gasior, M.: A ketogenic diet may offer neuroprotection in glaucoma and mitochondrial diseases of the optic nerve. Med Hypothesis Discov Innov Ophthalmol 1, 45-49 (2012).

109. Kinouchi, R., Ishiko, S., Hanada, K., Hayashi, H., Mikami, D., Tani, T., Zenimaru, T., Kawai, M., Nakabayashi, S., Kinouchi, M., Yoshida, A.: A low meat diet increases the risk of open-angle glaucoma in women - The results of population-based, cross-sectional study in Japan. PLoS One 13, e0204955 (2018).

110. Wan, M. J., Daniel, S., Kassam, F., Mutti, G., Butty, Z., Kasner, O., Trope, G. E., Buys, Y. M.: Survey of complementary and alternative medicine use in glaucoma patients. J Glaucoma 21, 79-82 (2012).

111. Al Owaifeer, A. M., Al Taisan, A. A.: The role of diet in glaucoma: A review of the current evidence. Ophthalmol Ther 7, 19-31 (2018).

112. Shapiro, A., Shapiro, Y., Udassin, R., Shoenfeld, Y., Konikoff, F.: The effect of salt loading diet on the intraocular pressure. Acta Ophthalmol (Copenh) 60, 35-40 (1982).

113. Ramdas, W. D., Wolfs, R. C., Kiefte-de Jong, J. C., Hofman, A., de Jong, P. T., Vingerling, J. R., Jansonius, N. M.: Nutrient intake and risk of open-angle glaucoma: The Rotterdam Study. Eur J Epidemiol 27, 385-393 (2012).

114. Giaconi, J. A., Yu, F., Stone, K. L., Pedula, K. L., Ensrud, K. E., Cauley, J. A., Hochberg, M. C., Coleman, A. L., Study of Osteoporotic Fractures Research Group: The association of consumption of fruits/vegetables with decreased risk of glaucoma among older African-American women in the study of osteoporotic fractures. Am J Ophthalmol 154, 635-644 (2012).

115. Coleman, A. L., Stone, K. L., Kodjebacheva, G., Yu, F., Pedula, K. L., Ensrud, K. E., Cauley, J. A., Hochberg, M. C., Topouzis, F., Badala, F., Mangione, C. M., Study of Osteoporotic Fractures Research Group: Glaucoma risk and the consumption of fruits and vegetables among older women in the study of osteoporotic fractures. Am J Ophthalmol 145, 1081-1089 (2008).

116. Kang, J. H., Willett, W. C., Rosner, B. A., Buys, E., Wiggs, J. L., Pasquale, L. R.: Association of dietary nitrate intake with primary open-angle glaucoma: A prospective analysis from the nurses' health study and health professionals follow-up study. JAMA Ophthalmol 134, 294-303 (2016). 